\title{
Spectral asymptotics for Robin problems with a discontinuous coefficient
}

\author{
Gerd Grubb \\ Dedicated to the memory of M. Sh. Birman (1928-2009)
}

\begin{abstract}
The spectral behavior of the difference between the resolvents of two realizations $\widetilde{A}_{1}$ and $\widetilde{A}_{2}$ of a second-order strongly elliptic symmetric differential operator $A$, defined by different Robin conditions $v u=b_{1} \gamma_{0} u$ and $v u=b_{2} \gamma_{0} u$, can, in the case where all coefficients are $C^{\infty}$, be determined by use of a general result on singular Green operators found by the author in 1984. Here we treat the problem for nonsmooth $b_{i}$. Using a Kreln resolvent formula, we show that, if $b_{1}$ and $b_{2}$ are in $L_{\infty}$, then the $s$-numbers $s_{j}$ of $\left(\widetilde{A}_{1}-\lambda\right)^{-1}-\left(\widetilde{A}_{2}-\lambda\right)^{-1}$ satisfy $s_{j} j^{3 /(n-1)} \leq C$ for all $j$; this improves a recent result for $A=-\Delta$ by Behrndt et al., that $\sum_{j} s_{j}^{p}<\infty$ for $p>(n-1) / 3$. A sharper estimate is obtained when $b_{1}$ and $b_{2}$ are in $C^{\varepsilon}$ for some $\varepsilon>0$, with jumps at a smooth hypersurface, namely that $s_{j} j^{3 /(n-1)} \rightarrow c$ for $j \rightarrow \infty$, with a constant $c$ defined from the principal symbol of $A$ and $b_{2}-b_{1}$.

As an auxiliary result we show that the usual principal spectral asymptotic estimate for pseudodifferential operators of negative order on a closed manifold extends to products of pseudodifferential operators interspersed with piecewise continuous functions.
\end{abstract}

Mathematics Subject Classification (2010). 35J40, 47G30, 58C40.

Keywords. Elliptic boundary value problem, Robin condition, spectral asymptotics, resolvent difference, Kreĭn formula, piecewise continuous coefficient, pseudodifferential boundary operator.

\section{Introduction}

Consider a second-order strongly elliptic symmetric operator

$$
A=-\sum_{j, k=1}^{n} \partial_{j}\left(a_{j k} \partial_{k} u\right)+a_{0} u
$$

on a bounded smooth domain $\Omega \subset \mathbb{R}^{n}$, and denote by $A_{\gamma}, A_{v}$, resp. $\tilde{A}$, the realizations in $L_{2}(\Omega)$ defined by the Dirichlet condition $\gamma_{0} u=0$, the Neumann condition $v u=0$, 
resp. a Robin condition $v u-b \gamma_{0} u=0$ with $b$ real. Here $\gamma_{0} u=\left.u\right|_{\partial \Omega}$, and

$$
\nu u=\sum_{j, k=1}^{n} n_{j} \gamma_{0}\left(a_{j k} \partial_{k} u\right),
$$

the conormal derivative, with $\vec{n}=\left(n_{1}, \ldots, n_{n}\right)$ denoting the interior normal to $\partial \Omega$. It is a classical result of Birman [5], shown also for exterior domains, that the difference between the resolvents of the Robin realization and the Dirichlet realization is compact and has the spectral behavior, for large negative $\lambda$,

$$
s_{j}\left((\tilde{A}-\lambda)^{-1}-\left(A_{\gamma}-\lambda\right)^{-1}\right) j^{2 /(n-1)} \leq C \quad \text { for all } j ;
$$

here $s_{j}(T)$ denotes the $j$-th eigenvalue of $\left(T^{*} T\right)^{1 / 2}$ (the $j$-th $s$-number or singular value of $T$ ), counted with multiplicities. This was shown assuming merely that $b \in L_{\infty}(\partial \Omega)$.

For the situation where all coefficients are $C^{\infty}$, the estimate was later improved to an asymptotic estimate

$$
s_{j}\left((\tilde{A}-\lambda)^{-1}-\left(A_{\gamma}-\lambda\right)^{-1}\right) j^{2 /(n-1)} \rightarrow c \quad \text { for } j \rightarrow \infty ;
$$

this follows from Grubb [18], Sect. 8 (with generalizations to higher-order operators), and Birman and Solomyak [7] (including exterior domains).

The paper [19] gave tools to extend (1.4) to nonselfadjoint situations (also for exterior domains by a cutoff technique), by showing that for any singular Green operator $G$ on $\Omega$ of order $-t<0$ and class 0 ,

$$
s_{j}(G) j^{t /(n-1)} \rightarrow c\left(g^{0}\right) \text { for } j \rightarrow \infty ;
$$

here $G$ belongs to the calculus of pseudodifferential boundary operators, introduced by Boutet de Monvel [8] and further developed in [19], [20]; $c\left(g^{0}\right)$ is a constant derived from the principal symbol $g^{0}$. In fact, the resolvent difference in (1.4) is a singular Green operator of order -2 and class 0 , when all coefficients are $C^{\infty}$.

Considering another resolvent difference, J. Behrndt, M. Langer, I. Lobanov, V. Lotoreichik, and I. Popov showed in a recent paper [4], on the basis of a theory of quasi-boundary triples by J. Behrndt and M. Langer [2], that, when $A=-\Delta$ (hence $v u=\gamma_{1} u=\sum_{j} n_{j} \gamma_{0} \partial_{j} u$ ) and $b$ is a real function in $L_{\infty}(\partial \Omega)$, the difference between the resolvent of $\widetilde{A}$ and the resolvent of the Neumann realization $A_{\nu}$ satisfies an estimate with 2 replaced by 3 , for $\lambda$ in the intersection of resolvent sets $\varrho(\widetilde{A}) \cap \varrho\left(A_{v}\right)$ :

$$
(\tilde{A}-\lambda)^{-1}-\left(A_{v}-\lambda\right)^{-1} \in \mathcal{C}_{p} \quad \text { for } p>3 /(n-1) ;
$$

here $\ell_{p}$ denotes the space of compact operators $T$ with singular value sequences $\left(s_{j}(T)\right)_{j \in \mathbb{N}} \in \ell_{p}$; the Schatten class of order $p$. (Besides real $b$, also functions with a fixed sign on $\operatorname{Im} b$ were treated.) 
In the case where $b \in C^{\infty}(\partial \Omega)$, this follows from (1.5), since $(\tilde{A}-\lambda)^{-1}-$ $\left(A_{v}-\lambda\right)^{-1}$ is a singular Green operator of order -3 , leading to the stronger estimate:

$$
s_{j}\left((\tilde{A}-\lambda)^{-1}-\left(A_{v}-\lambda\right)^{-1}\right) j^{3 /(n-1)} \rightarrow c \text { for } j \rightarrow \infty ;
$$

this was noted also in [24], Corollary 8.4 and Example 8.5.

The result of [4] is more general by treating nonsmooth $b$. Their main result Theorem 3.5 is proved in a formulation where the boundary condition is $\gamma_{0} u=\Theta \gamma_{1} u$ for an operator $\Theta$, but it is explained in their Remark 3.7 how this can be made to include general conditions $\gamma_{1} u=b \gamma_{0} u$ by use of the relations point of view of [2]; more on this in [3].

The main purpose of the present paper is to show spectral asymptotics estimates as in (1.7) for nonsmooth $b$. First we show (in Theorem 3.2) that an upper bound

$$
s_{j}\left((\tilde{A}-\lambda)^{-1}-\left(A_{v}-\lambda\right)^{-1}\right) j^{3 /(n-1)} \leq C \quad \text { for all } j
$$

holds for any complex $b \in L_{\infty}(\partial \Omega)$; this implies (1.6), for general $A$ as in (1.1). As a corollary, a similar estimate holds for $\left(\widetilde{A}_{1}-\lambda\right)^{-1}-\left(\widetilde{A}_{2}-\lambda\right)^{-1}$, when the $\widetilde{A}_{i}$ are defined by boundary conditions $v u=b_{i} \gamma_{0} u$ with $b_{i} \in L_{\infty}(\partial \Omega)$.

Next, we show (in Theorems 3.5 and 4.4) that asymptotic estimates hold when the $b_{i}$ are piecewise slightly better that continuous. Since asymptotic estimates are not additive, we must aim directly for $\left(\widetilde{A}_{1}-\lambda\right)^{-1}-\left(\widetilde{A}_{2}-\lambda\right)^{-1}$.

Theorem 1.1. Assume that $b_{1}, b_{2}$ and $b_{2}-b_{1}$ are piecewise $C^{\varepsilon}$ on $\partial \Omega$ for some $\varepsilon>0$, having jumps at $C^{\infty}$ hypersurfaces. Then

$$
\left.s_{j}\left(\left(\tilde{A}_{1}-\lambda\right)^{-1}-\left(\tilde{A_{2}}-\lambda\right)^{-1}\right)\right) j^{3 /(n-1)} \rightarrow c \text { for } j \rightarrow \infty,
$$

where $c$ is defined from the principal symbol of $A$ and $b_{2}-b_{1}$.

It suffices in fact that $b_{1}, b_{2}$ and $b_{2}-b_{1}$ are piecewise in $H_{p}^{r}(\partial \Omega)$ for some $r>0$ and $p>(n-1) / r$, see the details below.

For the proof of (1.8) the method is, as in [4], an application of functional analysis, building on a general theory of extensions (here Grubb [16]) together with known facts on elliptic boundary value problems. The proof of (1.9) in the nonsmooth situations draws on methods and results for pseudodifferential boundary operators in [19] and a result on restricted kernels of pseudodifferential operators by Laptev [27, 28].

As an auxiliary result of independent interest we show (Theorem 4.3) that a product of classical pseudodifferential operators of negative order on a closed manifold, interspersed with piecewise continuous functions having jumps at a smooth hypersurface, has a principal spectral asymptotics estimate as in the smooth case. Moreover, we extend (1.4) to $b \in L_{\infty}(\partial \Omega)$ (Theorem 3.4).

Some spectral estimates for resolvent differences in interior and exterior domains have been described recently by Malamud in [30], and spectral asymptotics have been shown in [23]; both papers treat higher-order operators but do not aim for the special 
bounds obtained here. Let us also mention that we do not here address the question of nonsmooth domains, as e.g. in Gesztesy and Mitrea [12], [13], [14] and Abels, Grubb, and Wood [1], [21], and their references.

To keep the paper short, some introductory material found in other sources will not be repeated here.

The main details of the extension theory [16]-[18] have been recalled and explained in several recent papers [9], [21], [24]; resulting Krěn-type resolvent formulas are shown in [9].

Sobolev spaces are recalled in numerous places. The basic facts we shall need on these and other function spaces such as Besov and Bessel-potential spaces, are recalled e.g. in [1], Sect. 2.

The calculus of pseudodifferential boundary operators is explained in Boutet de Monvel [8] and in [19], [20], [22].

\section{The Robin realization}

Let $\Omega$ be a bounded smooth subset of $\mathbb{R}^{n}$ with boundary $\partial \Omega=\Sigma$, and let

$$
a(u, v)=\sum_{j, k=1}^{n}\left(a_{j k} \partial_{k} u, \partial_{j} v\right)+\left(a_{0} u, v\right),
$$

be a sesquilinear form with coefficients in $C^{\infty}(\bar{\Omega})$ such that the associated secondorder operator (1.1) is formally selfadjoint and strongly elliptic. We assume moreover that $a(u, u)$ is real for $u \in H^{1}(\Omega)$ and (with $c>0, k \geq 0$ )

$$
a(u, u) \geq c\|u\|_{1}^{2}-k\|u\|_{0}^{2} \quad \text { for } u \in H^{1}(\Omega) .
$$

This holds if the matrix $\left(a_{j k}(x)\right)_{j, k=1}^{n}$ is real, symmetric, and positive definite and $a_{0}(x)$ is real, at each $x \in \bar{\Omega}$.

Let $b \in L_{\infty}(\Sigma)$, and define the sesquilinear form $a_{b}$ by

$$
a_{b}(u, v)=a(u, v)+\left(b \gamma_{0} u, \gamma_{0} v\right)_{L_{2}(\Sigma)} .
$$

Since $\left\|\gamma_{0} u\right\|_{L_{2}(\Sigma)}^{2} \leq c^{\prime}\|u\|_{3 / 4}^{2} \leq \varepsilon\|u\|_{1}^{2}+C(\varepsilon)\|u\|_{0}^{2}$ for any $\varepsilon$, we infer from (2.2) that

$$
\operatorname{Re} a_{b}(u, u) \geq c_{1}\|u\|_{1}^{2}-k_{1}\|u\|_{0}^{2} \quad \text { for } u \in H^{1}(\Omega),
$$

where $c_{1}<c$ is close to $c$ and $k_{1} \geq k$ is a large constant.

The sesquilinear form $a_{b}$ on $V=H^{1}(\Omega)$ in $H=L_{2}(\Omega)$ defines a realization $\widetilde{A}$ of $A$ by Lions' version of the Lax-Milgram lemma (as recalled e.g. in [22], Chapter 12), with domain

$$
D(\widetilde{A})=\left\{u \in H^{1}(\Omega) \cap D\left(A_{\max }\right) \mid(A u, v)=a_{b}(u, v) \text { for all } v \in H^{1}(\Omega)\right\} .
$$


The operator $\tilde{A}$ is closed, densely defined with spectrum in a sectorial region in $\left\{\operatorname{Re} \lambda \geq-k_{1}\right\}$, and its adjoint $\widetilde{A}^{*}$ is the analogous operator defined from

$$
a_{b}^{*}(u, v)=\overline{a(v, u)}+\left(\bar{b} \gamma_{0} u, \gamma_{0} v\right)_{L_{2}(\Sigma)} .
$$

In particular, when $b$ is real, $\tilde{A}$ is selfadjoint.

It will be useful to observe:

Lemma 2.1. For any small $\theta>0$ there is an $\alpha \geq 0$ such that the spectrum of $\tilde{A}$ is contained in the region

$$
M_{\theta, \alpha, k_{1}}=\left\{z \in \mathbb{C}|| \operatorname{Im} z \mid \leq \theta(\operatorname{Re} z+\alpha), \operatorname{Re} z \geq-k_{1}\right\} .
$$

Proof. Let $K=\|\operatorname{Im} b\|_{L_{\infty}(\Sigma)}$. From the inequalities for $a_{b}(u, u)$ we see that for $u \in H^{1}(\Omega)$,

$$
\begin{aligned}
\left|\operatorname{Im} a_{b}(u, u)\right| & =\left|\operatorname{Im}\left(b \gamma_{0} u, \gamma_{0} u\right)\right| \leq K\left(\varepsilon\|u\|_{1}^{2}+C(\varepsilon)\|u\|_{0}^{2}\right) \\
& \leq K \varepsilon c_{1}^{-1}\left(\operatorname{Re} a_{b}(u, u)+k_{1}\|u\|_{0}^{2}\right)+K C(\varepsilon)\|u\|_{0}^{2} \\
& =K \varepsilon c_{1}^{-1} \operatorname{Re} a_{b}(u, u)+\left(K \varepsilon c_{1}^{-1} k_{1}+K C(\varepsilon)\right)\|u\|_{0}^{2}
\end{aligned}
$$

This, together with (2.4), shows that for $u \neq 0, a_{b}(u, u) /\|u\|_{0}^{2}$ has its values in $M_{\theta, \alpha, k_{1}}$, where $\theta=K \varepsilon c_{1}^{-1}$ can be taken arbitrarily small, $\alpha=K \varepsilon c_{1}^{-1} k_{1}+K C(\varepsilon)$. The numerical ranges of $\tilde{A}$ and $\widetilde{A}^{*}$ are contained in this set, which then also contains the spectra. (More details for this kind of argument can be found in [22], Section 12.4.)

The Neumann-type boundary operator (1.2) enters in the "halfways Green's formula"

$$
(A u, v)-a(u, v)=\left(v u, \gamma_{0} v\right)_{L_{2}(\Sigma)},
$$

for smooth $u$ and $v$. It is known e.g. from [29] that $\gamma_{1}$ and $v$ extend to continuous mappings from $H^{1}(\Omega) \cap D\left(A_{\max }\right)$ to $H^{-1 / 2}(\Sigma)$, such that for $u \in H^{1}(\Omega) \cap D\left(A_{\max }\right)$, $v \in H^{1}(\Omega)$, formula (2.8) holds with the scalar product over $\Sigma$ replaced by the sesquilinear duality between $H^{-1 / 2}(\Sigma)$ and $H^{1 / 2}(\Sigma)$. Then

$$
(A u, v)-a_{b}(u, v)=\left(v u, \gamma_{0} v\right)_{H^{-1 / 2}(\Sigma), H^{1 / 2}(\Sigma)}-\left(b \gamma_{0} u, \gamma_{0} u\right)_{L_{2}(\Sigma)}
$$

and hence

$$
D(\tilde{A})=\left\{u \in H^{1}(\Omega) \cap D\left(A_{\max }\right) \mid v u=b \gamma_{0} u \text { in } H^{-1 / 2}(\Sigma)\right\},
$$

representing the Robin condition $v u-b \gamma_{0} u=0$.

For $b=0$, the condition is $v u=0$, defining what we call the Neumann realization $A_{v}$; it is selfadjoint with $D\left(A_{v}\right) \subset H^{2}(\Omega)$. It is well-known that when $b$ is smooth, then $D(\widetilde{A}) \subset H^{2}(\Sigma)$. 
Lemma 2.2. When $b \in L_{\infty}(\Sigma)$, the domain of $\tilde{A}$ satisfies

$$
D(\tilde{A}) \subset H^{3 / 2}(\Omega) \cap D\left(A_{\max }\right) .
$$

Proof. When $u \in D(\tilde{A})$, then $u \in H^{1}(\Omega)$ implies $\gamma_{0} u \in H^{1 / 2}(\Sigma) \subset L_{2}(\Sigma)$. Multiplication by $b$ is continuous on $L_{2}(\Sigma)$, so $b \gamma_{0} u \in L_{2}(\Sigma)$. Then also $v u=$ $b \gamma_{0} u$ is in $L_{2}(\Sigma)$. By the ellipticity of the Neumann problem, $A u \in L_{2}(\Omega)$ with $v u \in L_{2}(\Sigma)$ imply $u \in H^{3 / 2}(\Omega)$.

When $b$ has some smoothness or piecewise smoothness, we can get more regularity. It is known that when $b$ is in the Bessel potential space $H_{p}^{r}(\Sigma)$ with $r>(n-1) / p$, $p \geq 2$, then multiplication by $b$ is continuous in $H^{s}(\Sigma)$ for $|s| \leq r$ (cf e.g. Johnsen [26]). In relation to Hölder spaces $C^{r}$ and Besov spaces $B_{p . q}^{r}$ there are inclusions

$$
C^{r+2 \delta}(\Sigma) \hookrightarrow B_{\infty, 2}^{r+\delta}(\Sigma) \hookrightarrow B_{p, 2}^{r+\delta}(\Sigma) \hookrightarrow H_{p}^{r}(\Sigma) \quad \text { for any } \delta>0,
$$

so also functions in these spaces preserve $H^{s}(\Sigma)$ for $|s| \leq r$. (A summary of the relevant facts on function spaces is given e.g. in [1], Section 2.) Note that any $\varepsilon>0$ can be included as an $r+2 \delta$ by taking $r \in] 0, \varepsilon[, \delta=(\varepsilon-r) / 2$ and $p>(n-1) / r$.

When $X(\Sigma)$ is a function space over $\Sigma$, we say that $b$ is piecewise in $X$, when the $(n-1)$-dimensional manifold $\Sigma$ is a union $\Sigma_{1} \cup \cdots \cup \Sigma_{J}$ of smooth subsets $\Sigma_{j}$ with disjoint interiors (such that the interfaces are smooth $(n-2)$-dimensional manifolds), and there are functions $b_{j} \in X(\Sigma)$, such that $b$ equals $b_{j}$ on the interior $\Sigma_{j}^{\circ}$, for $j=1, \ldots, J$.

It is well known that multiplication by $1_{\Sigma_{j}}$ is continuous on $H^{s}(\Sigma)$ for all $|s|<$ $1 / 2$.

Proposition 2.3. (i) Let $b \in H_{p}^{r}(\Sigma)$ with $r>(n-1) / p, p \geq 2$ (it holds if $b$ is in one of the spaces in (2.10)). Then $D(\widetilde{A}) \subset H^{3 / 2+r}(\Omega)$ if $r<1 / 2, D(\widetilde{A}) \subset H^{2}(\Omega)$ if $r \geq 1 / 2$.

(ii) Let $b$ be piecewise in $H_{p}^{r}(\Sigma)$ with $r>(n-1) / p, p \geq 2$. Then $D(\widetilde{A}) \subset$ $H^{3 / 2+r}(\Omega)$ if $r<1 / 2, D(\widetilde{A}) \subset H^{2-\varepsilon}(\Omega)$ for any $\varepsilon>0$ if $r \geq 1 / 2$.

Proof. As already noted, $u \in H^{1}(\Omega)$ implies $\gamma_{0} u \in H^{1 / 2}(\Sigma)$. In the case (i), multiplication by $b$ preserves $H^{s}(\Sigma)$ for $|s| \leq r$, so $b \gamma_{0} u \in H^{\min \{r, 1 / 2\}}(\Sigma)$. Then also $v u=b \gamma_{0} u$ is in $H^{\min \{r, 1 / 2\}}(\Sigma)$, and now $A u \in L_{2}(\Omega)$ with $v u \in H^{\min \{r, 1 / 2\}}(\Sigma)$ imply $u \in H^{3 / 2+r}(\Omega)$ if $r<1 / 2, u \in H^{2}(\Omega)$ if $r \geq 1 / 2$, by the ellipticity of the Neumann problem.

In the case (ii), since $b=\sum_{j=1}^{J} b_{j} 1_{\Sigma_{j}}$, multiplication by $b$ maps $H^{r}(\Sigma)$ into itself if $r<1 / 2$, and into $H^{1 / 2-\varepsilon}$, any $\varepsilon>0$, if $r \geq 1 / 2$. Completing the proof as under (i), we find that $u \in H^{3 / 2+r}(\Omega)$ if $r<1 / 2, u \in H^{2-\varepsilon}(\Omega)$ if $r \geq 1 / 2$.

Let us regard $\tilde{A}$ from the point of view of the general extension theory of [16], as recalled in [9], [21], [24]. 
We take the Dirichlet realization $A_{\gamma}$ as the reference operator, assumed to have a positive lower bound. (Seen from the point of view of [16], [2] uses instead the Neumann realization $A_{v}$ as the reference operator.) The operator $\widetilde{A}$ corresponds, by the general theory of [16], to a closed densely defined operator $T: V \rightarrow W$, where $V$ and $W$ are closed subsets of $Z=\operatorname{ker} A_{\max }$, and $D(T)$ is dense in $V$; and this in turn is carried over by use of the homeomorphism $\gamma_{0}: Z \stackrel{\sim}{\longrightarrow} H^{-1 / 2}(\Sigma)$, to a closed operator $L: X \rightarrow Y^{*}$, with domain $D(L)$ dense in $X$, where $X$ and $Y$ are closed subspaces of $H^{-1 / 2}(\Sigma)$. Here $X=\gamma_{0} V, Y=\gamma_{0} W$, and $D(L)=\gamma_{0} D(T)=\gamma_{0} D(\widetilde{A})$.

Proposition 2.4. The operator $L: X \rightarrow Y$ corresponding to $\tilde{A}$ by [16] has $X=$ $Y=H^{-1 / 2}(\Sigma)$, and acts like $b-P_{\gamma, v}^{0}$ with a domain contained in $H^{1}(\Sigma)$. When $b$ is real, $L$ is selfadjoint as an unbounded operator from $H^{-1 / 2}(\Sigma)$ to $H^{1 / 2}(\Sigma)$.

Proof. Besides the description referred to above, we shall use the observations on operators defined by sesquilinear forms worked out in [17] (and partly recalled in [22], Chapter 13.2, see in particular Theorem 13.19). Since the domain of $a_{b}(u, v)$ equals $H^{1}(\Omega), T$ is defined from a sesquilinear form $t(z, w)$ with domain $H^{1}(\Omega) \cap Z$ dense in $Z$, and hence $V=W=Z$. It follows that $X=Y=H^{-1 / 2}(\Sigma)$, and $L$ is densely defined and closed as an operator from $H^{-1 / 2}(\Sigma)$ to $H^{1 / 2}(\Sigma)$. The adjoint $L^{*}$ is of the same type and corresponds to $\widetilde{A}^{*}$. When $b$ is real, $\widetilde{A}$ is selfadjoint as noted above; then $L$ is selfadjoint.

In the interpretation of the extension theory, $\tilde{A}$ represents the boundary condition

$$
\gamma_{0} u \in D(L), \quad \Gamma u=L \gamma_{0} u
$$

where $\Gamma u=v u-P_{\gamma, \nu}^{0} \gamma_{0} u$, so $L \gamma_{0} u=v u-P_{\gamma, \nu}^{0} \gamma_{0} u$ when $u \in D(\widetilde{A}) .\left(P_{\gamma, v}^{\lambda}\right.$ is the operator mapping Dirichlet boundary values to Neumann boundary values for solutions of $(A-\lambda) u=0$; more on this below.) Since the functions in $D(\widetilde{A})$ also satisfy $v u=b \gamma_{0} u$, we see that $L$ acts like

$$
L \varphi=\left(b-P_{\gamma, v}^{0}\right) \varphi
$$

By Lemma 2.2, $D(\tilde{A}) \subset H^{3 / 2}(\Omega)$, so $D(L)=\gamma_{0} D(\tilde{A}) \subset H^{1}(\Sigma)$.

When we replace $A$ by $A-\lambda$, where $\lambda$ is in the resolvent set $\varrho\left(A_{\gamma}\right)$ of $A_{\gamma}$, we get for the corresponding operator $L^{\lambda}$ :

$$
L^{\lambda} \text { acts like } b-P_{\gamma, \nu}^{\lambda}, \quad \text { with } D\left(L^{\lambda}\right)=D(L) \subset H^{1}(\Sigma) \text {. }
$$

For $\lambda \in \varrho\left(A_{\gamma}\right) \cap \varrho(\widetilde{A})$, there holds a Krel̆n resolvent formula (shown in [9], Theorem 3.4):

$$
(\tilde{A}-\lambda)^{-1}=\left(A_{\gamma}-\lambda\right)^{-1}+K_{\gamma}^{\lambda}\left(L^{\lambda}\right)^{-1}\left(K_{\gamma}^{\bar{\lambda}}\right)^{*}
$$


Here $K_{\gamma}^{\lambda}$ is the Poisson operator for the Dirichlet problem, i.e. the solution operator $K_{\gamma}^{\lambda}: \varphi \mapsto u$ for the problem

$$
(A-\lambda) u=0 \text { on } \Omega, \quad \gamma_{0} u=\varphi \text { on } \Sigma
$$

it maps $H^{s-1 / 2}(\Sigma) \rightarrow H^{s}(\Omega)$ continuously for all $s$, and the adjoint $\left(K_{\gamma}^{\lambda}\right)^{*}$ maps e.g. $L_{2}(\Omega)$ to $H^{1 / 2}(\Sigma)$.

We can use this to show a spectral estimate for $(\tilde{A}-\lambda)^{-1}-\left(A_{v}-\lambda\right)^{-1}$, going via differences with the Dirichlet resolvent. The argumentation is not the same as that of [4], which uses a Kreĭn formula based on the Poisson operator for the Neumann problem.

The spectrum of $A_{\gamma}$ is contained in a positive halfline $\left[c_{0}, \infty[\right.$, and the spectrum of $A_{v}$ is contained in a larger halfline ] $-k, \infty[$, $\operatorname{cf}(2.2)$. For $\lambda \in \mathbb{C} \backslash]-k, \infty[$, the Dirichlet-to-Neumann operator $P_{\gamma, v}^{\lambda}=v K_{\gamma}^{\lambda}$ is a homeomorphism from $H^{s}(\Sigma)$ to $H^{s-1}(\Sigma)$ for all $s \in \mathbb{R}$, with inverse $P_{v, \gamma}^{\lambda}$, the Neumann-to-Dirichlet operator. Then we can write

$$
L^{\lambda} \varphi=\left(b-P_{\gamma, \nu}^{\lambda}\right) \varphi=\left(b P_{\nu, \gamma}^{\lambda}-1\right) P_{\gamma, \nu}^{\lambda} \varphi, \quad \text { for } \varphi \in D(L) .
$$

Since $P_{v, \gamma}^{\lambda}$ is of order -1 , it is compact in $L_{2}(\Sigma)$. Then $b P_{v, \gamma}^{\lambda}-1$ is a Fredholm operator in $L_{2}(\Sigma)$, as noted also in [4]. If $\lambda$ is such that: (1) $L^{\lambda}$ is invertible (from $D(L)$ to $H^{1 / 2}(\Sigma)$ ), (2) $b P_{\nu, \gamma}^{\lambda}-1$ is invertible in $L_{2}(\Sigma)$, then the inverse of $L^{\lambda}$ must coincide with the inverse of $\left(b P_{\nu, \gamma}^{\lambda}-1\right) P_{\gamma, \nu}^{\lambda}$ on $H^{1 / 2}(\Sigma)$.

For $b P_{v, \gamma}^{\lambda}-1$, we get invertibility as follows, We have as a simple application of the principles in [20] ( $\mathrm{cf}$ Theorem 2.5.6, (A.25-26)) that

$$
\left\|P_{\gamma, \nu}^{\lambda} \varphi\right\|_{H^{s, \mu}(\Sigma)} \simeq\|\varphi\|_{H^{s+1, \mu}(\Sigma)}, \quad\|\varphi\|_{H^{s-1, \mu}(\Sigma)} \simeq\left\|P_{\nu, \gamma}^{\lambda} \varphi\right\|_{H^{s, \mu}(\Sigma)},
$$

uniformly in $\mu=|\lambda|^{1 / 2}$ for $\lambda \rightarrow \infty$ on rays in $\mathbb{C} \backslash \mathbb{R}_{+}$; this holds since $P_{\gamma, \nu}^{\lambda}$ is parameter-elliptic of order 1 and regularity $+\infty$ on the rays in $\mathbb{C} \backslash \mathbb{R}_{+}$. In particular, one has on such a ray $\left\{\lambda=\mu^{2} e^{i \eta}\right\}$ with $\left.\eta \in\right] 0,2 \pi[$, for $s \in[0,1]$ and $\mu \geq 1$,

$$
\left\|P_{\nu, \gamma}^{\lambda} \varphi\right\|_{H^{s}(\Sigma)}+\langle\mu\rangle^{s}\left\|P_{\nu, \gamma}^{\lambda} \varphi\right\|_{L_{2}(\Sigma)} \leq C \min \left\{\|\varphi\|_{H^{s-1}(\Sigma)},\langle\mu\rangle^{s-1}\|\varphi\|_{L_{2}(\Sigma)}\right\},
$$

so the norm of $P_{v, \gamma}^{\lambda}$ in $L_{2}(\Sigma)$ is $O\left(\langle\mu\rangle^{-1}\right)$ on the ray. Take $\mu_{0}$ so large that $\left\|b P_{v, \gamma}^{\lambda}\right\|_{\mathscr{L}\left(L_{2}(\Sigma)\right)} \leq \delta<1$ for $\mu \geq \mu_{0}$, then $b P_{v, \gamma}^{\lambda}-1$ is invertible as an operator in $L_{2}(\Sigma)$ for $\mu \geq \mu_{0}$, with a bounded inverse $\left(b P_{\nu, \gamma}^{\lambda}-1\right)^{-1}$ :

$$
\left(b P_{v, \gamma}^{\lambda}-1\right)^{-1}=-1-\sum_{k=1}^{\infty}\left(b P_{v, \gamma}^{\lambda}\right)^{k}, \quad \text { converging in } \mathscr{L}\left(L_{2}(\Sigma)\right) .
$$

Then $b-P_{\gamma, \nu}^{\lambda}$ has an inverse

$$
\left(b-P_{\gamma, \nu}^{\lambda}\right)^{-1}=P_{\nu, \gamma}^{\lambda}\left(b P_{\nu, \gamma}^{\lambda}-1\right)^{-1} .
$$


For $L^{\lambda}$ we know from the extension theory that $L^{\lambda}$ is bijective from $D(L)$ to $H^{1 / 2}(\Sigma)$ if and only if $\lambda \in \varrho(\widetilde{A})$. It follows from Lemma 1.1 by a simple geometric consideration that for each ray $\left\{\lambda=\mu^{2} e^{i \eta}\right\}$ with $\left.\eta \in\right] 0,2 \pi\left[\right.$, there is a $\mu_{1}$ such that such that $\lambda \in \varrho(\widetilde{A})$ for $\mu \geq \mu_{1}$.

For $\mu \geq \max \left\{\mu_{0}, \mu_{1}\right\}$, both (1) and (2) are satisfied, so then

$$
\left(L^{\lambda}\right)^{-1}=\left(b-P_{\gamma, v}^{\lambda}\right)^{-1}=P_{\nu, \gamma}^{\lambda}\left(b P_{\nu, \gamma}^{\lambda}-1\right)^{-1} \quad \text { on } H^{1 / 2}(\Sigma) .
$$

We note in particular that

$$
D\left(L^{\lambda}\right)=\left\{\varphi \in H^{1}(\Sigma) \mid\left(b-P_{\gamma, v}^{\lambda}\right) \varphi \in H^{1 / 2}(\Sigma)\right\},
$$

for such $\lambda$. Now $D(L)=D\left(L^{\lambda}\right)$, and $P_{\gamma, \nu}^{0}-P_{\gamma, \nu}^{\lambda}$ is bounded from $H^{-1 / 2}(\Sigma)$ to $H^{1 / 2}(\Sigma)$ (cf [9], Remark 3.2), so we conclude that

$$
D(L)=\left\{\varphi \in H^{1}(\Sigma) \mid\left(b-P_{\gamma, v}^{0}\right) \varphi \in H^{1 / 2}(\Sigma)\right\} .
$$

It follows moreover that (2.16) holds for all $\lambda \in \varrho\left(A_{\gamma}\right)$.

This shows the main part of:

Theorem 2.5. The domain of $L$ satisfies (2.17), and it is also described by (2.16) for any $\lambda \in \varrho\left(A_{\gamma}\right)$.

On each ray in $\mathbb{C} \backslash \mathbb{R}_{+}, \lambda$ is in $\varrho(\widetilde{A})$ and (2.15) holds for $|\lambda|$ sufficiently large. For such $\lambda$,

$$
(\tilde{A}-\lambda)^{-1}-\left(A_{\gamma}-\lambda\right)^{-1}=K_{\gamma}^{\lambda} P_{\nu, \gamma}^{\lambda}\left(b P_{\nu, \gamma}^{\lambda}-1\right)^{-1}\left(K_{\gamma}^{\bar{\lambda}}\right)^{*} .
$$

Proof. The statements before formula (2.18) were accounted for above, and the formula follows by insertion of (2.15) in (2.11).

\section{Spectral estimates}

Spectral estimates for resolvent differences will now be studied. A classical reference for the basic concepts is the book of Gohberg and Krenn [15]; some particularly relevant facts were collected in [19], supplied with additional results. We shall include a short summary here:

For $p>0$, the space $\ell_{p}$ is the Schatten class of compact linear operators $T$ (in a Hilbert space $H$ ) with singular value sequences $\left(s_{j}(T)\right)_{j \in \mathbb{N}} \in \ell_{p}$, and $\mathfrak{S}_{p}$ denotes the quasi-normed space of compact operators $T$ with $s_{j}(T)=O\left(j^{-1 / p}\right)$; here $\mathfrak{S}_{p} \subset \mathcal{C}_{p+\varepsilon}$ for all $\varepsilon>0$.

The rules shown by Ky Fan [11]

$$
s_{j+k-1}\left(T+T^{\prime}\right) \leq s_{j}(T)+s_{k}\left(T^{\prime}\right), \quad s_{j+k-1}\left(T T^{\prime}\right) \leq s_{j}(T) s_{k}\left(T^{\prime}\right),
$$


imply that $\ell_{p}$ and $\mathfrak{S}_{p}$ are vector spaces, and that a product rule holds:

$$
\mathfrak{S}_{p} \cdot \mathfrak{S}_{q} \subset \mathfrak{S}_{1 /\left(p^{-1}+q^{-1}\right)}, \quad \ell_{p} \cdot \ell_{q} \subset \ell_{1 /\left(p^{-1}+q^{-1}\right)} .
$$

Moreover, the rule

$$
s_{j}(A T B) \leq\|A\| s_{j}(T)\|B\|
$$

implies that $\mathfrak{S}_{p}$ and $\mathcal{C}_{p}$ are preserved under compositions with bounded operators. We mention two perturbation results:

Lemma 3.1. (i) If $s_{j}(T) j^{1 / p} \rightarrow C_{0}$ and $s_{j}\left(T^{\prime}\right) j^{1 / p} \rightarrow 0$ for $j \rightarrow \infty$, then $s_{j}\left(T+T^{\prime}\right) j^{1 / p} \rightarrow C_{0}$ for $j \rightarrow \infty$.

(ii) If $T=T_{M}+T_{M}^{\prime}$ for each $M \in \mathbb{N}$, where $s_{j}\left(T_{M}\right) j^{1 / p} \rightarrow C_{M}$ for $j \rightarrow \infty$ and $s_{j}\left(T_{M}^{\prime}\right) j^{1 / p} \leq \varepsilon_{M}$ for $j \in \mathbb{N}$, with $C_{M} \rightarrow C_{0}$ and $\varepsilon_{M} \rightarrow 0$ for $M \rightarrow \infty$, then $s_{j}(T) j^{1 / p} \rightarrow C_{0}$ for $j \rightarrow \infty$.

The statement in (i) is the Weyl-Ky Fan theorem (cf e.g. [15] Theorem II 2.3), and (ii) is a refinement shown in [19], Lemma 4.2.2 .

We also recall that when $\Xi$ is a compact $n^{\prime}$-dimensional smooth manifold (possibly with boundary) and $T$ is a bounded linear operator from $L_{2}(\Xi)$ to $H^{t}(\Xi)$ for some $t>0$, then $T \in \mathfrak{S}_{n^{\prime} / t}$ as an operator in $L_{2}(\Xi)$, with

$$
s_{j}(T) j^{t / n^{\prime}} \leq C\|T\|_{\mathscr{L}\left(L_{2}, H^{t}\right)},
$$

$C$ depending only on $\Xi$ and $t$. See [19], Lemma 4.4ff. for references.

The Poisson operator $K_{\gamma}^{\lambda}$ is continuous from $H^{s-1 / 2}(\Sigma)$ to $H^{s}(\Omega)$ for all $s \in \mathbb{R}$, and its adjoint $K_{\gamma}^{\lambda^{*}}$ is a trace operator of class 0 and order -1 in the pseudodifferential boundary operator calculus, hence is continuous from $H^{s}(\Omega)$ to $H^{s+1 / 2}(\Sigma)$ for $s>-1 / 2$. Then the composition $K_{\gamma}^{\lambda^{*}} K_{\gamma}^{\lambda}$ is continuous from $L^{2}(\Sigma)$ to $H^{1}(\Sigma)$, so in view of (3.4), $K_{\gamma}^{\lambda^{*}} K_{\gamma}^{\lambda} \in \mathfrak{S}_{n-1}$ and hence $K_{\gamma}^{\lambda} \in \mathfrak{S}_{(n-1) /(1 / 2)}$, as operators in $L_{2}(\Sigma)$. The singular numbers of $K_{\gamma}^{\lambda^{*}}$ have the same behavior. Moreover, since $P_{\nu, \gamma}^{\lambda}$ is a pseudodifferential operator of order -1 on $\Sigma$, it lies in $\mathfrak{S}_{n-1}$ when considered as an operator in $L_{2}(\Sigma)$.

Theorem 3.2. Let $b \in L_{\infty}(\Sigma)$. For any $\lambda \in \varrho(\widetilde{A}) \cap \varrho\left(A_{v}\right)$,

$$
(\tilde{A}-\lambda)^{-1}-\left(A_{v}-\lambda\right)^{-1} \in \mathfrak{S}_{(n-1) / 3} .
$$

Proof. First assume that $\lambda$ lies so far out on a ray in $\mathbb{C} \backslash \mathbb{R}_{+}$that the statements in Theorem 2.5 are valid. 
Applying (2.18) to our $\tilde{A}$ and also to the case $b=0$ (the Neumann realization), we find by subtraction:

$$
\begin{aligned}
(\tilde{A}-\lambda)^{-1}-\left(A_{v}-\lambda\right)^{-1}= & (\tilde{A}-\lambda)^{-1}-\left(A_{\gamma}-\lambda\right)^{-1} \\
& -\left(\left(A_{\nu}-\lambda\right)^{-1}-\left(A_{\gamma}-\lambda\right)^{-1}\right) \\
= & K_{\gamma}^{\lambda} P_{\nu, \gamma}^{\lambda}\left[\left(b P_{\nu, \gamma}^{\lambda}-1\right)^{-1}+1\right] K_{\gamma}^{\bar{\lambda}^{*}} \\
= & K_{\gamma}^{\lambda} P_{\nu, \gamma}^{\lambda}\left(b P_{\nu, \gamma}^{\lambda}-1\right)^{-1} b P_{\nu, \gamma}^{\lambda} K_{\gamma}^{\bar{\lambda}^{*}} .
\end{aligned}
$$

The last expression is composed of the operator $K_{\gamma}^{\lambda}$ in $\mathfrak{S}_{(n-1) /(1 / 2)}$, the adjoint of $K_{\gamma}^{\bar{\lambda}}$ with the same property, two factors $P_{v, \gamma}^{\lambda}$ in $\mathfrak{S}_{n-1}$ and the bounded operators $\left(b P_{v, \gamma}^{\lambda}-1\right)^{-1}$ and $b$, so it belongs to $\mathfrak{S}_{(n-1) / 3}$, by (3.2).

Now let $\lambda^{\prime}$ be an arbitrary number in $\varrho(\widetilde{A}) \cap \varrho\left(A_{v}\right)$. We use the following refined resolvent identity as in [4]:

$$
\begin{aligned}
\left(S-\lambda^{\prime}\right)^{-1}-\left(T-\lambda^{\prime}\right)^{-1}=(1+ & \left.\left(\lambda^{\prime}-\lambda\right)\left(T-\lambda^{\prime}\right)^{-1}\right) \\
& \left((S-\lambda)^{-1}-(T-\lambda)^{-1}\right) \\
& \left(1+\left(\lambda^{\prime}-\lambda\right)\left(S-\lambda^{\prime}\right)^{-1}\right),
\end{aligned}
$$

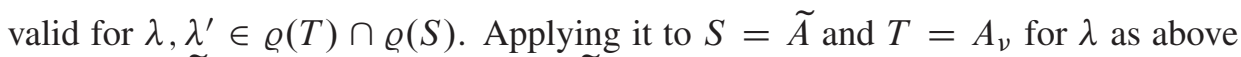
and $\lambda^{\prime} \in \varrho(\widetilde{A}) \cap \varrho\left(A_{v}\right)$, we find that $\left(\widetilde{A}-\lambda^{\prime}\right)^{-1}-\left(A_{\nu}-\lambda^{\prime}\right)^{-1}$ is a composition of an operator in $\mathfrak{S}_{(n-1) / 3}$ with two bounded operators, hence lies in $\mathfrak{S}_{(n-1) / 3}$, as was to be shown.

There is an obvious corollary:

Corollary 3.3. Let $b_{1}, b_{2} \in L_{\infty}(\Sigma)$, and denote the corresponding realizations of Robin conditions $v u=b_{1} \gamma_{0} u$ resp. $v u=b_{2} \gamma_{0} u$ by $\widetilde{A}_{1}$ resp. $\widetilde{A}_{2}$. For any $\lambda \in \varrho\left(\widetilde{A}_{1}\right) \cap \varrho\left(\widetilde{A}_{2}\right)$,

$$
\left(\widetilde{A_{1}}-\lambda\right)^{-1}-\left(\tilde{A}_{2}-\lambda\right)^{-1} \in \mathfrak{S}_{(n-1) / 3} .
$$

Proof. Write $\left(\tilde{A}_{1}-\lambda\right)^{-1}-\left(\tilde{A}_{2}-\lambda\right)^{-1}$ as the difference between $\left(\tilde{A}_{1}-\lambda\right)^{-1}-$ $\left(A_{v}-\lambda\right)^{-1}$ and $\left(\tilde{A}_{2}-\lambda\right)^{-1}-\left(A_{\gamma}-\lambda\right)^{-1}$, then the result follows from Theorem 3.2, and (3.7), since $\mathfrak{S}_{p}$ is a vector space.

Formula (2.18) also allows us to show a spectral asymptotics estimate for $(\tilde{A}-\lambda)^{-1}$ $-\left(A_{\gamma}-\lambda\right)^{-1}$ that was obtained in the smooth case for selfadjoint realizations and negative $\lambda$ in Grubb [18], Section 8, and Birman and Solomyak [7]. In the former paper it is shown, also for $2 m$-order problems, that the operator is, on the orthogonal complement of its nullspace, isometric to an elliptic pseudodifferential operator on $\Sigma$ of order $-2 m$ (which has the asserted spectral asymptotics); in the latter paper exterior domains are included. 
Theorem 3.4. Let $b \in L_{\infty}(\Sigma)$. For any $\lambda \in \varrho(\widetilde{A}) \cap \varrho\left(A_{\gamma}\right)$,

$$
s_{j}\left((\tilde{A}-\lambda)^{-1}-\left(A_{\gamma}-\lambda\right)^{-1}\right) j^{2 /(n-1)} \rightarrow C_{0}^{2 /(n-1)} \text { for } j \rightarrow \infty,
$$

where $C_{0}$ is the same constant as in the case $b=0$ (where $\left.\tilde{A}=A_{v}\right)$, namely

$$
C_{0}=\frac{1}{(n-1)(2 \pi)^{n-1}} \int_{\Sigma} \int_{\left|\xi^{\prime}\right|=1}\left(\left\|\tilde{k}^{0}\right\|_{L_{2}\left(\mathbb{R}_{+}\right)}\left|p^{0}\right|^{1 / 2}\right)^{n-1} d \omega\left(\xi^{\prime}\right) d x^{\prime} ;
$$

here $\tilde{k}^{0}\left(x^{\prime}, x_{n}, \xi^{\prime}\right)$ is the principal symbol-kernel of $K_{\gamma}^{\lambda}$ and $p^{0}\left(x^{\prime}, \xi^{\prime}\right)$ is the principal symbol of $P_{v, \gamma}^{\lambda}$.

Proof. Since the details are perhaps not very well known, we first give a proof of (3.9)-(3.10) in the case $b=0$. We have as an easy special case of (2.18) that

$$
\left(A_{v}-\lambda\right)^{-1}-\left(A_{\gamma}-\lambda\right)^{-1}=-K_{\gamma}^{\lambda} P_{v, \gamma}^{\lambda} K_{\gamma}^{\bar{\lambda}^{*}} \equiv G_{\nu}
$$

This is a singular Green operator with principal boundary symbol operator

$$
g_{v}^{0}\left(x^{\prime}, \xi^{\prime}, D_{n}\right)=-k^{0}\left(x^{\prime}, \xi^{\prime}, D_{n}\right) p^{0}\left(x^{\prime}, \xi^{\prime}\right) k^{0}\left(x^{\prime}, \xi^{\prime}, D_{n}\right)^{*}
$$

in local coordinates, where $k^{0}$ and $p^{0}$ are the ( $\lambda$-independent) principal symbols of $K_{\gamma}^{\lambda}$ and $P_{v, \gamma}^{\lambda}$. At each $\left(x^{\prime}, \xi^{\prime}\right), k^{0}\left(x^{\prime}, \xi^{\prime}, D_{n}\right): \mathbb{C} \rightarrow L_{2}\left(\mathbb{R}_{+}\right)$maps $v \in \mathbb{C}$ to $\tilde{k}^{0}\left(x^{\prime}, x_{n}, \xi^{\prime}\right) v$, where $\tilde{k}^{0}\left(x^{\prime}, x_{n}, \xi^{\prime}\right) \in S\left(\bar{R}_{+}\right)$is the symbol-kernel. In the case $A=-\Delta$ it equals $e^{-\left|\xi^{\prime}\right| x_{n}}$, and it has a similar structure for general $A$ (cf e.g. [25], Section 2.d). The operator $k^{0}\left(x^{\prime}, \xi^{\prime}, D_{n}\right)^{*}: L_{2}\left(\mathbb{R}_{+}\right) \rightarrow \mathbb{C}$ maps $u\left(x_{n}\right)$ to $\left(u, \tilde{k}^{0}\right)_{L_{2}\left(\mathbb{R}_{+}\right)}$. Thus $k^{0}\left(x^{\prime}, \xi^{\prime}, D_{n}\right)^{*} k^{0}\left(x^{\prime}, \xi^{\prime}, D_{n}\right)$ is the multiplication by $\left\|\tilde{k}^{0}\right\|_{L_{2}\left(\mathbb{R}_{+}\right)}^{2}$, and thus $k^{0}\left(x^{\prime}, \xi^{\prime}, D_{n}\right) k^{0}\left(x^{\prime}, \xi^{\prime}, D_{n}\right)^{*}$ is the rank 1 operator mapping $u$ to $\left(u, \tilde{k}^{0}\right) \tilde{k}^{0}$. The latter operator has the sole eigenvector $\tilde{k}_{1}^{0}=\tilde{k}^{0} /\left\|\tilde{k}^{0}\right\|$ with a positive eigenvalue $\left\|\tilde{k}^{0}\right\|^{2}$ (besides eigenvectors in the nullspace), so its trace equals the eigenvalue. The middle factor $p^{0}$ is just multiplication by a scalar; for $A=-\Delta$, it equals $-\left|\xi^{\prime}\right|^{-1}$.

By [19], Theorem 4.10, since $G_{v}$ is a singular Green operator of order -2 and class 0 ,

$$
s_{j}\left(G_{v}\right) j^{2 /(n-1)} \rightarrow C\left(g_{v}^{0}\right)^{2 /(n-1)} \text { for } j \rightarrow \infty
$$

where

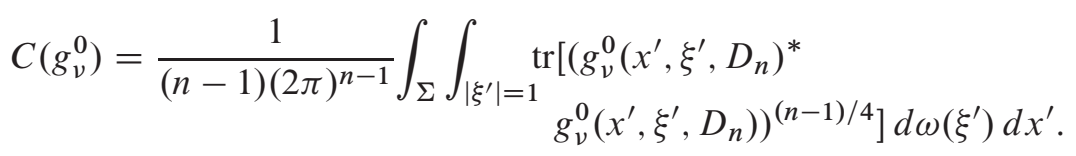

Here

$$
\begin{array}{r}
g_{v}^{0}\left(x^{\prime}, \xi^{\prime}, D_{n}\right)^{*} g_{v}^{0}\left(x^{\prime}, \xi^{\prime}, D_{n}\right)=k^{0}\left(x^{\prime}, \xi^{\prime}, D_{n}\right) \bar{p}^{0}\left(x^{\prime}, \xi^{\prime}\right) k^{0}\left(x^{\prime}, \xi^{\prime}, D_{n}\right)^{*} \\
k^{0}\left(x^{\prime}, \xi^{\prime}, D_{n}\right) p^{0}\left(x^{\prime}, \xi^{\prime}\right) k^{0}\left(x^{\prime}, \xi^{\prime}, D_{n}\right)^{*}
\end{array}
$$




$$
\begin{array}{r}
=\left\|\tilde{k}^{0}\left(x^{\prime}, x_{n}, \xi^{\prime}\right)\right\|_{L_{2}\left(\mathbb{R}_{+}\right)}^{2}\left|p^{0}\left(x^{\prime}, \xi^{\prime}\right)\right|^{2} \\
k^{0}\left(x^{\prime}, \xi^{\prime}, D_{n}\right) k^{0}\left(x^{\prime}, \xi^{\prime}, D_{n}\right)^{*} .
\end{array}
$$

This is a rank 1 operator with eigenvalue $\left\|\tilde{k}^{0}\right\|_{L_{2}}^{4}\left|p^{0}\right|^{2}$, so

$$
\operatorname{tr}\left[\left(g_{v}^{0}\left(x^{\prime}, \xi^{\prime}, D_{n}\right)^{*} g_{v}^{0}\left(x^{\prime}, \xi^{\prime}, D_{n}\right)\right)^{(n-1) / 4}\right]=\left(\left\|\tilde{k}^{0}\right\|_{L_{2}}\left|p^{0}\right|^{1 / 2}\right)^{n-1},
$$

and (3.10) follows.

Now the case of general $b$. For large $\lambda$ on rays in $\mathbb{C} \backslash \mathbb{R}+$ as in Theorem 2.5 we write formula (2.13) as

$$
\left(b P_{\nu, \gamma}^{\lambda}-1\right)^{-1}=-1-b P_{\nu, \gamma}^{\lambda} S, \quad \text { where } S=\sum_{k=0}^{\infty}\left(b P_{\nu, \gamma}^{\lambda}\right)^{k} \in \mathscr{L}\left(L_{2}(\Sigma)\right) \text {. }
$$

Then we have from (2.18):

$$
\begin{aligned}
(\tilde{A}-\lambda)^{-1}-\left(A_{\gamma}-\lambda\right)^{-1} & =K_{\gamma}^{\lambda} P_{\nu, \gamma}^{\lambda}\left(-1-b P_{\nu, \gamma}^{\lambda} S\right) K_{\gamma}^{\bar{\lambda}^{*}} \\
& =-K_{\gamma}^{\lambda} P_{\nu, \gamma}^{\lambda} K_{\gamma}^{\bar{\lambda}^{*}}-K_{\gamma}^{\lambda} P_{\nu, \gamma}^{\lambda} b P_{\nu, \gamma}^{\lambda} S K_{\gamma}^{\bar{\lambda}^{*}} .
\end{aligned}
$$

The first term equals $\left(A_{\nu}-\lambda\right)^{-1}-\left(A_{\gamma}-\lambda\right)^{-1}$ and satisfies the spectral asymptotics estimate (3.9) with (3.10). The second term is in $\mathfrak{S}_{(n-1) / 3}$, in view of the mapping properties of its factors, as in the proof of Theorem 3.2. By Lemma 3.1 (i), it follows that the sum of the two terms has the asymptotic behavior (3.9).

General $\lambda \in \varrho(\widetilde{A}) \cap \varrho\left(A_{\gamma}\right)$ are included by use of the resolvent identity (3.7), which gives the operator as a sum of a term with the behavior (3.9) and terms in $\mathfrak{S}_{(n-1) /(2+t)}$ with $t>0$, using that $\left(A_{\gamma}-\lambda\right)^{-1} \in \mathfrak{S}_{n / 2}$ and $(\widetilde{A}-\lambda)^{-1} \in \mathfrak{S}_{n /(3 / 2)}$. Then Lemma 3.1 (i) applies to show (3.9) for the sum.

Spectral asymptotics estimates for the resolvent difference (3.5) are harder to get at, since $b$ here enters in the principal part of the operator. However, with a little smoothness of $b$ we can obtain the spectral estimate by reduction to a case that allows an approximation procedure.

We consider the resolvent difference of two general Robin problems from the start, since the asymptotic property is not in general additive.

Theorem 3.5. Assume that $b_{1}, b_{2} \in H_{p}^{r}(\Sigma)$, where $r>0$ and $p>(n-1) / r, p \geq 2$; this holds if the $b_{i}$ are in one of the spaces in (2.10). Define $\widetilde{A}_{i}$ as in Corollary 3.3. Then for $\lambda \in \varrho\left(\widetilde{A_{1}}\right) \cap \varrho\left(\widetilde{A}_{2}\right)$,

$$
s_{j}\left(\left(\tilde{A_{1}}-\lambda\right)^{-1}-\left(\tilde{A_{2}}-\lambda\right)^{-1}\right) j^{3 /(n-1)} \rightarrow C\left(g^{0}\right)^{3 /(n-1)} \text { for } j \rightarrow \infty,
$$

where

$$
\begin{aligned}
& C\left(g^{0}\right)=\frac{1}{(n-1)(2 \pi)^{n-1}} \int_{\Sigma} \int_{\left|\xi^{\prime}\right|=1}\left(\left\|\tilde{k}^{0}\right\|_{L_{2}\left(\mathbb{R}_{+}\right)}^{2}\left|p^{0}\right|^{2}\right. \\
& \left.\left|b_{2}-b_{1}\right|\right)^{(n-1) / 3} d \omega\left(\xi^{\prime}\right) d x^{\prime} .
\end{aligned}
$$


Proof. First let $\lambda$ be large on a ray in $\mathbb{C} \backslash \mathbb{R}_{+}$such that Theorem 2.5 applies to $\widetilde{A}_{1}$ and $\widetilde{A}_{2}$. Using (3.14) in the form

$$
\left(b_{i} P_{\nu, \gamma}^{\lambda}-1\right)^{-1}=-1-b_{i} P_{\nu, \gamma}^{\lambda}-\left(b_{i} P_{\nu, \gamma}^{\lambda}\right)^{2} S_{i}
$$

we have that

$$
\left(b_{1} P_{v, \gamma}^{\lambda}-1\right)^{-1}-\left(b_{2} P_{v, \gamma}^{\lambda}-1\right)^{-1}=\left(b_{2}-b_{1}\right) P_{v, \gamma}^{\lambda}-\left(b_{1} P_{v, \gamma}^{\lambda}\right)^{2} S_{1}-\left(b_{2} P_{v, \gamma}^{\lambda}\right)^{2} S_{2} \text {. }
$$

Then we get, using (3.6),

$$
\begin{gathered}
\left(\tilde{A}_{1}-\lambda\right)^{-1}-\left(\tilde{A}_{2}-\lambda\right)^{-1}=\left(\tilde{A}_{1}-\lambda\right)^{-1}-\left(A_{v}-\lambda\right)^{-1} \\
-\left(\left(\tilde{A}_{2}-\lambda\right)^{-1}-\left(A_{\nu}-\lambda\right)^{-1}\right) \\
=K_{\gamma}^{\lambda} P_{\nu, \gamma}^{\lambda}\left[\left(b_{1} P_{\nu, \gamma}^{\lambda}-1\right)^{-1}+1\right] K_{\gamma}^{\bar{\lambda}^{*}} \\
-K_{\gamma}^{\lambda} P_{\nu, \gamma}^{\lambda}\left[\left(b_{2} P_{\nu, \gamma}^{\lambda}-1\right)^{-1}+1\right] K_{\gamma}^{\bar{\lambda}^{*}} \\
=K_{\gamma}^{\lambda} P_{\nu, \gamma}^{\lambda}\left(b_{2}-b_{1}\right) P_{\nu, \gamma}^{\lambda} K_{\gamma}^{\bar{\lambda}^{*}} \\
-K_{\gamma}^{\lambda} P_{\nu, \gamma}^{\lambda}\left(b_{1} P_{\nu, \gamma}^{\lambda}\right)^{2} S_{1} K_{\gamma}^{\bar{\lambda}^{*}} \\
\quad+K_{\gamma}^{\lambda} P_{\nu, \gamma}^{\lambda}\left(b_{2} P_{\nu, \gamma}^{\lambda}\right)^{2} S_{2} K_{\gamma}^{\bar{\lambda}^{*}} \\
=G+F_{1}+F_{2} .
\end{gathered}
$$

In the terms $F_{i}$ we use for one of the factors $b_{i} P_{\nu, \gamma}^{\lambda}$ that $b_{i}$ preserves $H^{s}(\Sigma)$ for $|s| \leq r$ (see the text before Proposition 2.3), so that $b_{i} P_{v, \gamma}^{\lambda}$ maps $L_{2}(\Sigma)$ continuously into $H^{r^{\prime}}(\Sigma), r^{\prime}=\min \{r, 1\}$. So this factor is in $\mathfrak{S}_{(n-1) / r^{\prime}}$, together with the usual two factors in $\mathfrak{S}_{(n-1) /(1 / 2)}$ and two factors in $\mathfrak{S}_{n-1}$, whereby the full composed operator $F_{i}$ is in $\mathfrak{S}_{(n-1) /\left(3+r^{\prime}\right)}$. It will not influence the spectral asymptotics.

In the term $G$, let us denote $b_{2}-b_{1}=b$. We write $b$ for each $M \in \mathbb{N}$ as a sum

$$
b=b_{M}+b_{M}^{\prime},
$$

where $b_{M} \in C^{\infty}(\Sigma)$ and $\sup _{x^{\prime} \in \Sigma}\left|b_{M}^{\prime}\left(x^{\prime}\right)\right| \leq 1 / M$; this is possible since $b$ is continuous on the smooth compact manifold $\Sigma$. Accordingly, we write $G=G_{M}+$ $G_{M}^{\prime}$ with

$$
G_{M}=K_{\gamma}^{\lambda} P_{\nu, \gamma}^{\lambda} b_{M} P_{\nu, \gamma}^{\lambda} K_{\gamma}^{\bar{\lambda}^{*}}, \quad G_{M}^{\prime}=K_{\gamma}^{\lambda} P_{\nu, \gamma}^{\lambda} b_{M}^{\prime} P_{\nu, \gamma}^{\lambda} K_{\gamma}^{\bar{\lambda}^{*}} .
$$

Here $G_{M}^{\prime}$ is a composition of fixed operators with the usual $\mathfrak{S}_{p}$-properties and a factor $b_{M}^{\prime}$ whose norm in $\mathscr{L}\left(L_{2}(\Sigma)\right)$ is $\leq 1 / M$; this implies that

$$
\sup _{j} s_{j}\left(G_{M}^{\prime}\right) j^{3 /(n-1)} \leq C / M \text { for all } M,
$$

for a suitable constant $C$, in view of (3.3). 
The term $G_{M}$ is treated by application of the tools in [19]. Since $b_{M} \in C^{\infty}, G_{M}$ is a genuine singular Green operator of order -3 and class 0 , with polyhomogeneous symbol. The principal symbol $g_{M}^{0}$ is the symbol of the boundary symbol operator (in local coordinates)

$$
\begin{aligned}
g_{M}^{0}\left(x^{\prime}, \xi^{\prime}, D_{n}\right)=k^{0}\left(x^{\prime}, \xi^{\prime}, D_{n}\right) p^{0}\left(x^{\prime}, \xi^{\prime}\right) b_{M}\left(x^{\prime}\right) \\
\\
p^{0}\left(x^{\prime}, \xi^{\prime}\right) k^{0}\left(x^{\prime}, \xi^{\prime}, D_{n}\right)^{*} .
\end{aligned}
$$

It follows from [19], Theorem 4.10, that

$$
s_{j}\left(G_{M}\right) j^{3 /(n-1)} \rightarrow C\left(g_{M}^{0}\right)^{3 /(n-1)} \text { for } j \rightarrow \infty,
$$

where

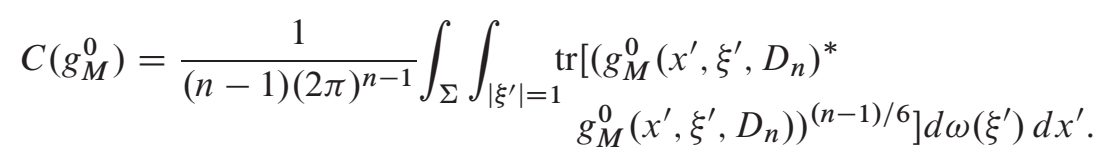

As in the analysis of $g_{v}{ }^{*} g_{v}^{0}$ in the proof of Theorem 3.4, now with the middle factor $p^{0}$ replaced by $p^{0} b_{M} p^{0}$, we find that

$$
\begin{aligned}
\operatorname{tr}\left[\left(g_{M}^{0}\left(x^{\prime}, \xi^{\prime}, D_{n}\right)^{*} g_{M}^{0}\left(x^{\prime}, \xi^{\prime}, D_{n}\right)\right)^{(n-1) / 6}\right] & =\left(\left\|\tilde{k}^{0}\right\|_{L_{2}}^{4}\left|p^{0}\right|^{4}\left|b_{M}\right|^{2}\right)^{(n-1) / 6} \\
& =\left(\left\|\tilde{k}^{0}\right\|_{L_{2}}^{2}\left|p^{0}\right|^{2}\left|b_{M}\right|\right)^{(n-1) / 3}
\end{aligned}
$$

and hence

$$
\begin{aligned}
& C\left(g_{M}^{0}\right)=\frac{1}{(n-1)(2 \pi)^{n-1}} \int_{\Sigma} \int_{\left|\xi^{\prime}\right|=1}\left(\left\|\tilde{k}^{0}\right\|_{L_{2}\left(\mathbb{R}_{+}\right)}^{2}\right. \\
& \left.\left|p^{0}\right|^{2}\left|b_{M}\right|\right)^{(n-1) / 3} d \omega\left(\xi^{\prime}\right) d x^{\prime} .
\end{aligned}
$$

When $M \rightarrow \infty, b_{M}\left(x^{\prime}\right) \rightarrow b\left(x^{\prime}\right)$ uniformly in $x^{\prime}$, so

$$
C\left(g_{M}^{0}\right) \rightarrow C\left(g^{0}\right)
$$

where

$$
C\left(g^{0}\right)=\frac{1}{(n-1)(2 \pi)^{n-1}} \int_{\Sigma} \int_{\left|\xi^{\prime}\right|=1}\left(\left\|\tilde{k}^{0}\right\|_{L_{2}\left(\mathbb{R}_{+}\right)}^{2}\left|p^{0}\right|^{2}|b|\right)^{(n-1) / 3} d \omega\left(\xi^{\prime}\right) d x^{\prime},
$$

with $b=b_{2}-b_{1}$.

Now we first apply Lemma 3.1 (ii) to the decompositions $G=G_{M}+G_{M}^{\prime}$; this shows that $G$ has the spectral behavior in (3.16). When $F_{1}$ and $F_{2}$ are added to $G$, we can use Lemma 3.1 (i) to conclude that also $G+F_{1}+F_{2}$ has the spectral behavior in (3.16).

Finally, general $\lambda \in \varrho(\widetilde{A}) \cap \varrho\left(A_{v}\right)$ are included by use of the resolvent formula (3.7) as in the preceding proof.

In the case $A=-\Delta$, where $\tilde{k}^{0}$ and $p^{0}$ are independent of $x^{\prime}$, the formula for $C\left(g^{0}\right)$ reduces to a constant times $\int_{\Sigma}\left|b_{2}-b_{1}\right|^{(n-1) / 3} d x^{\prime}$. 


\section{Coefficients with jumps}

It possible to extend the result of Theorem 3.5 to cases where $b$ has jump discontinuities by use of special results for pseudodifferential operators (from here on abbreviated to $\psi$ do's). In showing this, we also supply the general knowledge on spectral asymptotics for $\psi$ do's multiplied with nonsmooth functions.

Let $\Xi$ be a compact $n^{\prime}$-dimensional $C^{\infty}$-manifold without boundary, and assume that it is divided by a smooth $\left(n^{\prime}-1\right)$-dimensional hypersurface into two subsets $\Xi_{+}$ and $\Xi_{-}\left(n^{\prime}\right.$-dimensional $C^{\infty}$-manifolds with boundary) such that $\Xi=\Xi_{+} \cup \Xi_{-}$, $\Xi_{+}^{\circ} \cap \Xi_{-}^{\circ}=\emptyset, \partial \Xi_{+}=\partial \Xi_{-}$. (Since the sets need not be connected, this covers the situation of $J$ smooth subsets described before Proposition 2.3.) We denote by $r^{ \pm}$the restrictions from $\Xi$ to $\Xi_{ \pm}$, and by $e^{ \pm}$the extension-by-zero operators from functions on $\Xi_{ \pm}$to functions on $\Xi$ :

$$
e^{ \pm} u=\left\{\begin{array}{l}
u \text { on } \Xi_{ \pm} \\
0 \text { on } \Xi_{\mp}
\end{array} .\right.
$$

Multiplication by the characteristic function $1 \Xi_{+}$for $\Xi_{+}$can also be written $e^{+} r^{+}$; similarly $1 \Xi_{-}=e^{-} r^{-}$.

It is well-known (as recalled e.g. in [19], Lemma 4.5) that when $P$ is an $N \times N$ matrix formed classical $\psi$ do on $\Xi$ of negative order $-t$, then it satisfies the spectral asymptotics formulas for $j \rightarrow \infty$ :

$$
\begin{aligned}
s_{j}(P) j^{t / n^{\prime}} & \rightarrow C\left(p^{0}\right)^{t / n^{\prime}} \text { in general, } \\
\pm \lambda_{j}^{ \pm}(P) j^{t / n^{\prime}} & \rightarrow C^{ \pm}\left(p^{0}\right)^{t / n^{\prime}} \text { if } P \text { is selfadjoint, }
\end{aligned}
$$

where, respectively,

$$
\begin{aligned}
C\left(p^{0}\right) & =\frac{1}{n^{\prime}(2 \pi)^{n^{\prime}}} \int_{\Xi} \int_{|\xi|=1} \operatorname{tr}\left[\left(p^{0}(x, \xi)^{*} p^{0}(x, \xi)\right)^{n^{\prime} / 2 t}\right] d \omega(\xi) d x, \\
C^{ \pm}\left(p^{0}\right) & =\frac{1}{n^{\prime}(2 \pi)^{n^{\prime}}} \int_{\Xi} \int_{|\xi|=1} \sum_{\mathrm{ev} . \gtrless 0}\left( \pm \lambda_{j}^{ \pm}\left(p^{0}(x, \xi)\right)^{n^{\prime} / t}\right) d \omega(\xi) d x .
\end{aligned}
$$

Let us also recall the result of Laptev [27], [28]:

Proposition 4.1. Let $P$ be a classical pseudodifferential operator on $\Xi$ of negative order $-t$. Then $1 \Xi_{+} P 1 \Xi_{-} \in \mathfrak{S}_{\left(n^{\prime}-1\right) / t}$.

(Expressed in local coordinates, this means that the operator whose kernel is the restriction of the kernel of $P$ to the second or fourth quadrant, picks up the boundary dimension in its spectral behavior. For $\psi$ do's having the transmission property at $\partial \Xi_{+}$, this is confirmed by the results of [19].)

The rules in the following are valid also for $N \times N$-matrix formed operators $P$ and factors $b$, and would then need a trace indication tr in the integrals; we leave this aspect out here for simplicity. 
Theorem 4.2. Let $P$ be a classical pseudodifferential operator of negative order $-t$, such that $(P u, u) \geq 0$ for $u \in L_{2}(\Xi)$. Then $P_{(+)}=1 \Xi_{+} P 1_{\Xi_{+}}$satisfies the spectral asymptotics formula

$$
s_{j}\left(P_{(+)}\right) j^{t / n^{\prime}} \rightarrow c\left(P_{(+)}\right)^{t / n^{\prime}} \text { for } j \rightarrow \infty,
$$

where

$$
\begin{aligned}
c\left(P_{(+)}\right) & =\frac{1}{n^{\prime}(2 \pi)^{n^{\prime}}} \int_{\Xi_{+}} \int_{|\xi|=1}\left(p^{0}(x, \xi)^{*} p^{0}(x, \xi)\right)^{n^{\prime} / 2 t} d \omega(\xi) d x \\
& =\frac{1}{n^{\prime}(2 \pi)^{n^{\prime}}} \int_{\Xi_{+}} \int_{|\xi|=1} p^{0}(x, \xi)^{n^{\prime} / t} d \omega(\xi) d x .
\end{aligned}
$$

Proof. The principal symbol $p^{0}$ is $\geq 0$; which explains the second identity in (4.4). Introduce two $C^{\infty}$ cutoff functions $\zeta_{1}$ and $\zeta_{2}$ taking values in $[0,1]$ such that $\zeta_{1}=1$ on $\Xi_{+}$and vanishes outside a neighborhood of $\Xi_{+}$, and $\zeta_{2}=0$ on $\Xi_{-}$and is 1 outside a neighborhood of $\Xi_{-}$. We shall then compare $P_{(+)}$with the operators (all are compact in $\left.L_{2}(\Xi)\right)$

$$
P_{1}=\zeta_{1} P \zeta_{1} \quad \text { and } \quad P_{2}=\zeta_{2} P \zeta_{2}
$$

When $u \in L_{2}(\Xi)$, denote $e^{ \pm} r^{ \pm} u=u_{ \pm}$. We have for $P_{1}$, since $\zeta_{1} u_{+}=u_{+}$:

$$
\begin{aligned}
\left(P_{1} u, u\right) & =\left(P_{1} u_{+}, u_{+}\right)+\left(P_{1} u_{+}, u_{-}\right)+\left(P_{1} u_{-}, u_{+}\right)+\left(P_{1} u_{-}, u_{-}\right) \\
& =\left(P_{(+)} u, u\right)+(R u, u)+\left(P \zeta_{1} u_{-}, \zeta_{1} u_{-}\right),
\end{aligned}
$$

where $R=1_{\Xi_{-}} P_{1} I_{\Xi_{+}}+1_{\Xi_{+}} P_{1} I_{\Xi_{-}}$. Since $P_{1}$ is a classical $\psi$ do of order $-t$ on $\Xi$, it has the spectral behavior in (4.1)-(4.2) with the limit $C\left(p_{1}^{0}\right)^{t / n^{\prime}}$; here

$$
C\left(p_{1}^{0}\right)=\frac{1}{n^{\prime}(2 \pi)^{n^{\prime}}} \int_{\operatorname{supp} \zeta_{1}} \int_{|\xi|=1}\left(\zeta_{1} p^{0}(x, \xi) \zeta_{1}\right)^{n^{\prime} / t} d \omega(\xi) d x .
$$

Moreover, $R$ is of the type considered in Proposition 4.1, hence lies in $\mathfrak{S}_{\left(n^{\prime}-1\right) / t}$. Then by Lemma 3.1 (i), $P_{1}-R$ likewise has the spectral behavior in (4.1)-(4.2) with the limit $C\left(p_{1}^{0}\right)^{t / n^{\prime}}$. Now observe that, since $P$ is nonnegative, $\left(P \zeta_{1} u_{-}, \zeta_{1} u_{-}\right) \geq 0$ for all $u \in L_{2}(\Xi)$. Thus we have:

$$
\left(P_{(+)} u, u\right) \leq\left(\left(P_{1}-R\right) u, u\right), \quad \text { for all } u \in L_{2}(\Xi) .
$$

Both operators $P_{(+)}$and $P_{1}-R$ are selfadjoint nonnegative, so the $s$-numbers are the same as the eigenvalues, and the minimum-maximum principle implies in view of (4.5) that

$$
s_{j}\left(P_{(+)}\right) \leq s_{j}\left(P_{1}-R\right), \quad \text { for all } j .
$$

It then follows from the limit property of the $s_{j}\left(P_{1}-R\right)$ that

$$
\limsup _{j \rightarrow \infty} s_{j}\left(P_{(+)}\right) j^{t / n^{\prime}} \leq C\left(p_{1}^{0}\right)^{t / n^{\prime}} .
$$


For the comparison with $P_{2}$ we write, using that $\zeta_{2} u_{+}=\zeta_{2} u$,

$$
\begin{aligned}
\left(P_{(+)} u, u\right)= & \left(\zeta_{2} P \zeta_{2} u_{+}, u_{+}\right)+\left(\left(1-\zeta_{2}\right) P\left(1-\zeta_{2}\right) u_{+}, u_{+}\right) \\
& +\left(\left(1-\zeta_{2}\right) P \zeta_{2} u_{+}, u_{+}\right)+\left(\zeta_{2} P\left(1-\zeta_{2}\right) u_{+}, u_{+}\right) \\
\geq & \left(\zeta_{2} P \zeta_{2} u, u\right)+\left(\left(1-\zeta_{2}\right) P \zeta_{2} u, u_{+}\right)+\left(\zeta_{2} P\left(1-\zeta_{2}\right) u_{+}, u\right) \\
= & \left(\left(\zeta_{2} P \zeta_{2}+\left(1-\zeta_{2}\right) P \zeta_{2}+\zeta_{2} P\left(1-\zeta_{2}\right)\right) u, u\right)+\left(R_{1} u, u\right),
\end{aligned}
$$

where $R_{1}$ is a sum of terms as in Proposition 4.1. Then since $s_{j}\left(P_{(+)}\right)=\lambda_{j}\left(P_{(+)}\right) \geq$ $\lambda_{j}^{+}\left(\zeta_{2} P \zeta_{2}+\left(1-\zeta_{2}\right) P \zeta_{2}+\zeta_{2} P\left(1-\zeta_{2}\right)+R_{1}\right)$,

$$
\liminf _{j \rightarrow \infty} S_{j}\left(P_{(+)}\right) j^{t / n^{\prime}} \geq C^{+}\left(\zeta_{2} p^{0} \zeta_{2}+\left(1-\zeta_{2}\right) p^{0} \zeta_{2}+\zeta_{2} p^{0}\left(1-\zeta_{2}\right)\right)^{t / n^{\prime}} .
$$

Since $C\left(p_{1}^{0}\right)$ and $C^{+}\left(\zeta_{2} p^{0} \zeta_{2}+\left(1-\zeta_{2}\right) p^{0} \zeta_{2}+\zeta_{2} p^{0}\left(1-\zeta_{2}\right)\right)$ come arbitrarily close to $c\left(P_{(+)}\right)$when the support of $\zeta_{1}$ shrinks towards $\Xi_{+}$and the support of $1-\zeta_{2}$ shrinks towards $\Xi_{-}$, we conclude that (4.3) with (4.4) holds.

This leads to a result on compositions of $\psi$ do's with discontinuous factors, which seems to have an interest in itself:

Theorem 4.3. Let $P$ be an operator composed of $l$ classical pseudodifferential operators $P_{1}, \ldots, P_{l}$ of negative orders $-t_{1}, \ldots,-t_{l}$ and $l+1$ functions $b_{1}, \ldots, b_{l+1}$ that are piecewise continuous on $\Xi$ with possible jumps at $\partial \Xi_{+}$(so the $b_{k}$ extend to continuous functions on $\Xi_{+}$and on $\Xi_{-}$);

$$
P=b_{1} P_{1} \ldots b_{l} P_{l} b_{l+1} \text {. }
$$

Let $t=t_{1}+\cdots+t_{l}$. Then $P$ has the spectral behavior:

$$
s_{j}(P) j^{t / n^{\prime}} \rightarrow c(P)^{t / n^{\prime}} \text { for } j \rightarrow \infty
$$

where

$$
\begin{aligned}
c(P) & =\frac{1}{n^{\prime}(2 \pi)^{n^{\prime}}} \int_{\Xi} \int_{|\xi|=1}\left(\bar{b}_{l+1}(x) p_{l}^{0}(x, \xi)^{*} \ldots p_{1}^{0}(x, \xi)^{*} \bar{b}_{1}(x)\right. \\
& \left.b_{1}(x) p_{1}^{0}(x, \xi) \ldots p_{l}^{0}(x, \xi) b_{l+1}(x)\right)^{n^{\prime} / 2 t} d \omega(\xi) d x \\
= & \frac{1}{n^{\prime}(2 \pi)^{n^{\prime}}} \int_{\Xi} \int_{|\xi|=1}\left|b_{1} \ldots b_{l+1} p_{1}^{0} \ldots p_{l}^{0}\right|^{n^{\prime} / t} d \omega(\xi) d x .
\end{aligned}
$$

Proof. We can write

$$
\begin{aligned}
P^{*} P & =\bar{b}_{l+1} P_{l}^{*} \ldots P_{1}^{*} \bar{b}_{1} b_{1} P_{1} \ldots P_{l} b_{l} \\
& =1_{\Xi_{+}} P^{*} P 1_{\Xi_{+}}+1_{\Xi_{-}} P^{*} P 1_{\Xi_{-}}+R,
\end{aligned}
$$

where $R=1_{\Xi_{+}} \bar{b}_{l+1} P_{l}^{*} \ldots P_{l} b_{l} 1_{\Xi_{-}}+1_{\Xi_{-}} \bar{b}_{l+1} P_{l}^{*} \ldots P_{l} b_{l} 1_{\Xi_{+}}$. Inserting $1=$ $1_{\Xi_{+}}+1_{\Xi_{-}}$at each factor $b_{k}$ or $\bar{b}_{k}$ in $R$ and multiplying out, we obtain it as a sum of 
terms of order $-t$, each containing at least one factor of the type in Proposition 4.1. Thus $R \in \mathfrak{S}_{n^{\prime} /(t+\delta)}$ with a $\delta>0$. For the term $1_{\Xi_{+}} P^{*} P 1_{\Xi_{+}}$, we proceed as in Theorem 3.5. We can assume that $b_{k}$ is extended from $\Xi_{+}$to a continuous function $b_{k}$ on $\Xi$. Each $b_{k}$ is approximated by a uniformly convergent sequence $b_{k M}$ of $C^{\infty}$-functions on $\Xi$. For each $M$,

$$
P_{M}^{*} P_{M}=\bar{b}_{l+1, M} P_{l}^{*} \ldots P_{1}^{*} \bar{b}_{1 M} b_{1 M} P_{1} \ldots b_{l M} P_{l} b_{l+1, M}
$$

is a classical nonnegative $\psi$ do of order $-t$, so Theorem 4.2 applies to the operator with $1_{\Xi_{+}}$before and after, and gives the corresponding spectral asymptotics formula. Since $P_{M}^{*} P_{M}-P^{*} P$ can be written as a sum of terms where each has a small factor $b_{k M}-b_{k}$ or $\bar{b}_{k M}-\bar{b}_{k}$, we have for $M \rightarrow \infty$ that

$$
\sup _{j} s_{j}\left(1 \Xi_{+} P_{M}^{*} P_{M} 1 \Xi_{+}-1 \Xi_{+} P^{*} P 1_{\Xi_{+}}\right) j^{t / n^{\prime}} \rightarrow 0 .
$$

Then Lemma 3.1 (ii) implies a spectral asymptotics formula for $1 \Xi_{+} P^{*} P 1_{\Xi_{+}}$, with the constant as in (4.11) but integrated over $\Xi_{+}$. - There is a similar result for $1_{\Xi_{-}} P^{*} P 1_{\Xi_{-}}$, relative to $\Xi_{-}$.

Now since $L_{2}(\Xi)$ identifies with the orthogonal sum of $L_{2}\left(\Xi_{+}\right)$and $L_{2}\left(\Xi_{-}\right)$, the spectra are simply superposed when the operators are added together. The statement $\lambda_{j}(T) j^{t / n^{\prime}} \rightarrow c(T)^{t / n^{\prime}}$ for $j \rightarrow \infty$ is equivalent with $N^{\prime}(a ; T) a^{n^{\prime} / t} \rightarrow c(T)$ for $a \rightarrow \infty$, where $N^{\prime}(a ; T)$ counts the number of eigenvalues in $[1 / a, \infty[$; superposition of the spectra means addition of the counting functions. (More on counting functions e.g. in [20], Section A.6.) Thus $1_{\Xi_{+}} P^{*} P 1_{\Xi_{+}}+1_{\Xi_{-}} P^{*} P 1_{\Xi_{-}}$has a spectral asymptotics behavior where the constant is obtained by adding the integrals for $1_{\Xi_{+}} P^{*} P 1_{\Xi_{+}}$and $1_{\Xi_{-}} P^{*} P 1_{\Xi_{-}}$, so it is as described in (4.9)-(4.11). By Lemma 3.1 (i), the behavior keeps this form when we add $R$ to the operator.

A similar theorem holds for matrix formed operators $P_{k}$ and factors $b_{k}$, with $c(P)$ defined by the first expression in (4.11); here of course it cannot be reduced to the second expression unless all the factors commute.

A special case of the situation in Theorem 4.3 is the case of $b P$, where $P$ is a classical $\psi$ do and $b$ is a piecewise continuous function. We need a case with interspersed factors $b_{k}$ in our application below.

We can now show:

Theorem 4.4. The conclusion of Theorem 3.5 holds also when $b_{1}$ and $b_{2}$ are piecewise in $H_{p}^{r}(\Sigma)$ for some $r>0$ as in Theorem $3.5, b_{1}, b_{2}$, and $b_{2}-b_{1}$ having jumps at a smooth hypersurface.

Proof. We use again the decomposition in (3.18):

$$
\left(\tilde{A_{1}}-\lambda\right)^{-1}-\left(\tilde{A_{2}}-\lambda\right)^{-1}=G+F_{1}+F_{2},
$$




$$
\begin{aligned}
& \text { with } G=K_{\gamma}^{\lambda} P_{v, \gamma}^{\lambda}\left(b_{2}-b_{1}\right) P_{v, \gamma}^{\lambda} K_{\gamma}^{\bar{\lambda}^{*}}, \\
& \qquad \begin{array}{r}
F_{1}=-K_{\gamma}^{\lambda} P_{v, \gamma}^{\lambda}\left(b_{1} P_{\nu, \gamma}^{\lambda}\right)^{2} S_{1} K_{\gamma}^{\bar{\lambda}^{*}}, \\
F_{2}=K_{\gamma}^{\lambda} P_{v, \gamma}^{\lambda}\left(b_{2} P_{\nu, \gamma}^{\lambda}\right)^{2} S_{2} K_{\gamma}^{\bar{\lambda}^{*}}
\end{array}
\end{aligned}
$$

and $F_{1}$ and $F_{2}$ are handled as after (3.18), using that $b_{i} P_{v, \gamma}^{\lambda}$ maps $L_{2}(\Sigma)$ into $H^{r^{\prime}}(\Sigma)$, $r^{\prime}=\min \{r, 1 / 2-\varepsilon\}$. Then they are in $\mathfrak{S}_{(n-1) /\left(3+r^{\prime}\right)}$. We denote again $b_{2}-b_{1}=b$.

For $G$ we proceed as follows. Let $\lambda$ be large negative, so that Theorem 2.5 holds. Since $\lambda$ is real, $K_{\gamma}^{\bar{\lambda}}=K_{\gamma}^{\lambda}$, and $P_{\nu, \gamma}^{\lambda}$ is selfadjoint. The $j$-th eigenvalue of $G^{*} G$ satisfies

$$
\lambda_{j}\left(G^{*} G\right)=\lambda_{j}\left(K_{\gamma}^{\lambda} P_{\nu, \gamma}^{\lambda} \bar{b} P_{\nu, \gamma}^{\lambda} K_{\gamma}^{\lambda^{*}} K_{\gamma}^{\lambda} P_{v, \gamma}^{\lambda} b P_{v, \gamma}^{\lambda} K_{\gamma}^{\lambda^{*}}\right) .
$$

Here $K_{\gamma}^{\lambda^{*}} K_{\gamma}^{\lambda}$ equals a selfadjoint $\psi$ do $P_{1}$ of order -1 ; it is nonnegative on $L_{2}(\Sigma)$ and injective, since $K_{\gamma}^{\lambda}$ is injective:

$$
\left(P_{1} \varphi, \varphi\right)_{L_{2}(\Sigma)}=\left(K_{\gamma}^{\lambda^{*}} K_{\gamma}^{\lambda} \varphi, \varphi\right)_{L_{2}(\Sigma)}=\left\|K_{\gamma}^{\lambda} \varphi\right\|_{L_{2}(\Omega)}^{2} \geq c\|\varphi\|_{H^{-1 / 2}(\Sigma)}^{2},
$$

hence elliptic. It follows from Seeley [31] that $P_{1}$ has a squareroot $P_{2}=P_{1}^{1 / 2}$ which is a classical elliptic $\psi$ do of order $-1 / 2$. Then we find, applying the general formula

$$
\lambda_{j}\left(T T^{\prime}\right)=\lambda_{j}\left(T^{\prime} T\right)
$$

with $T=K_{\gamma}^{\lambda} P_{v, \gamma}^{\lambda} \bar{b} P_{v, \gamma}^{\lambda} P_{2}, T^{\prime}=P_{2} P_{v, \gamma}^{\lambda} b P_{v, \gamma}^{\lambda} K_{\gamma}^{\lambda^{*}}$, that

$$
\begin{aligned}
\lambda_{j}\left(G^{*} G\right) & =\lambda_{j}\left(K_{\gamma}^{\lambda} P_{\nu, \gamma}^{\lambda} \bar{b} P_{\nu, \gamma}^{\lambda} P_{2} P_{2} P_{\nu, \gamma}^{\lambda} b P_{\nu, \gamma}^{\lambda} K_{\gamma}^{\lambda^{*}}\right) \\
& =\lambda_{j}\left(P_{2} P_{\nu, \gamma}^{\lambda} b P_{\nu, \gamma}^{\lambda} K_{\gamma}^{\lambda^{*}} K_{\gamma}^{\lambda} P_{v, \gamma}^{\lambda} \bar{b} P_{\nu, \gamma}^{\lambda} P_{2}\right) \\
& =\lambda_{j}\left(P_{2} P_{\nu, \gamma}^{\lambda} b P_{\nu, \gamma}^{\lambda} P_{1} P_{\nu, \gamma}^{\lambda} \bar{b} P_{v, \gamma}^{\lambda} P_{2}\right) .
\end{aligned}
$$

The operator $Q=P_{2} P_{v, \gamma}^{\lambda} b P_{v, \gamma}^{\lambda} P_{1} P_{v, \gamma}^{\lambda} \bar{b} P_{v, \gamma}^{\lambda} P_{2}$ is an operator to which Theorem 4.3 applies, and it gives a spectral asymptotics formula with the constant defined as in (4.11), with $n^{\prime}=n-1$. Since $p_{1}^{0}=\left\|\tilde{k}^{0}\right\|_{L_{2}}^{2}, p_{2}^{0}=\left\|\tilde{k}^{0}\right\|_{L_{2}}$, the formula can be rewritten in the form (3.17).

The proof is now completed in the same way as in the proof of Theorem 4.5.

The results can be extended to exterior domains by the method of [23].

In a forthcoming paper we shall treat the question of spectral asymptotics for the mixed problem for $-\Delta+a_{0}$, where the boundary condition jumps from a Dirichlet condition to a Neumann condition at a smooth hypersurface of $\Sigma$. Here we moreover need to draw on the analyses of nonstandard pseudodifferential operators, as in Shamir [32], Eskin [10], Birman and Solomyak [6], and many later works. 
Added in proof. The paper on the mixed problem has appeared in J. Math. Analysis Appl. 382 (2011), 339-363 and needs the following corrections.

- Page 351, line 4 from below. Omit " $H^{1 / 2}\left(\Sigma_{+}^{\circ}\right) \subset^{\prime}$; replace " $H^{1}(\Sigma)$ " by " $L_{2}(\Sigma)$ ".

- Page 361, line 4. Replace "(Th. 3.3)" by “(Th. 4.3)".

\section{References}

[1] H. Abels, G. Grubb, and I. Wood, Extension theory and Krel̆n-type resolvent formulas for nonsmooth boundary value problems. Preprint 2010. arXiv:1008.3281

[2] J. Behrndt and M. Langer, Boundary value problems for elliptic partial differential operators on bounded domains. J. Funct. Anal. 243 (2007), 536-565. MR 2289696 Zbl 1132.47038

[3] J. Behrndt and M. Langer, Elliptic operators, Dirichlet-to-Neumann maps and quasi boundary triples. Preprint, for Proceedings of Leiden workshop 2009.

[4] J. Behrndt, M. Langer, I. Lobanov, V. Lotoreichik, and I. Popov, A remark on Schatten-von Neumann properties of resolvent differences of generalized Robin Laplacians on bounded domains. J. Math. Analysis Appl. 371 (2010), 750-758. MR 2670152 Zbl 1198.35159

[5] M. S. Birman, Perturbations of the continuous spectrum of a singular elliptic operator by varying the boundary and the boundary conditions. Vestnik Leningrad. Univ. 17 (1962), 22-55, English transl. by M. Z. Solomyak: T. Suslina and D. Yafaev (eds.), Spectral theory of differential operators. Amer. Math. Soc., Providence (RI), 2008, 19-53. MR 0138874 Zbl 1184.35234

[6] M. S. Birman and M. Z. Solomyak, Asymptotics of the spectrum of pseudo-differential operators with anisotropic-homogeneous symbols. Vestnik Leningrad Univ. 7 (1977), 1321. English transl.: Vestnik Leningrad Univ. Math. 10 (1982), 237-247. Zbl 0439.47040

[7] M. S. Birman and M. Z. Solomyak, Asymptotics of the spectrum of variational problems on solutions of elliptic equations in unbounded domains. Funkts. Analiz Prilozhen 14 (1980), 27-35. English transl.: Funct. Anal. Appl. 4 (1981), 267-274. MR 0595726 Zbl 0463.35061

[8] L. Boutet de Monvel, Boundary problems for pseudo-differential operators. Acta Math. 126 (1971), 11-51. MR 0407904 Zbl 0206.39401

[9] B. M. Brown, G. Grubb, and I. G. Wood, $M$-functions for closed extensions of adjoint pairs of operators with applications to elliptic boundary problems. Math. Nachr. 282 (2009), 314-347. MR 2503156 Zbl 1167.47057

[10] G. I. Eskin, Boundary value problems for elliptic pseudodifferential equations. Amer. Math. Soc., Providence (RI),1981. MR 0623608 Zbl 0458.35002

[11] Ky Fan, Maximum properties and inequalities for the eigenvalues of completely continuous operators. Proc. Nat. Acad. Sci. USA 37 (1951), 760-766. MR 0045952 Zbl 0044.11502 
[12] F. Gesztesy and M. Mitrea, Robin-to-Robin maps and Krel̆n-type resolvent formulas for Schrödinger operators on bounded Lipschitz domains. In M. L. Gorbachuk, V. Gorbachuk, A. N. Kochubei, H. Langer, and G. Popov (eds), Modern Analysis and Applications. The Mark Kreı̆n Centenary Conference, Vol. 2. Birkhäuser, Basel, 2009, 81-113. MR 2569392 Zbl 1180.35186

[13] F. Gesztesy and M. Mitrea, Nonlocal Robin Laplacians and some remarks on a paper by Filonov on eigenvalue inequalities. J. Differential Equations 247 (2009), 2871-2896. MR 2568160 Zbl 1181.35155

[14] F. Gesztesy and M. Mitrea, A description of all selfadjoint extensions of the Laplacian and Krěn-type resolvent formulas in nonsmooth domains. J. Analyse Math. 113 (2011), 53-172.

[15] I. C. Gohberg and M. G. Kreĭn, Introduction to the theory of linear nonselfadjoint operators. Amer. Math. Soc., Providence (RI), 1969. MR 0246142 Zbl 0181.13504

[16] G. Grubb, A characterization of the non-local boundary value problems associated with an elliptic operator. Ann. Scuola Norm. Sup. Pisa III 22 (1968), 425-513. MR 0239269 Zbl 0182.14501

[17] G. Grubb, Les problèmes aux limites généraux d'un opérateur elliptique, provenant de la théorie variationnelle. Bull. Sc. Math. 94 (1970), 113-157. MR 0280866 Zbl 0201.46502

[18] G. Grubb, Properties of normal boundary problems for elliptic even-order systems. Ann. Scuola Norm. Sup. Pisa IV 1 (1974), 1-61. MR 0492833 Zbl 0309.35034

[19] G. Grubb, Singular Green operators and their spectral asymptotics. Duke Math. J. 51 (1984), 477-528. MR 0757950 Zbl 0553.58034

[20] G. Grubb, Functional calculus of pseudodifferential boundary problems. Second Edition. Birkhäuser, Basel, 1996. MR 1385196 Zbl 0844.35002

[21] G. Grubb, KreĬn resolvent formulas for elliptic boundary problems in nonsmooth domains. Rend. Sem. Mat. Univ. Pol. Torino 66 (2010), 13-39. MR 2537622 Zbl 1206.35090

[22] G. Grubb, Distributions and operators. Springer Verlag, New York, 2009. MR 2453959 Zbl 1171.47001

[23] G. Grubb, Perturbation of essential spectra of exterior elliptic problems. Appl. Anal. 90 (2011), 103-123. Zbl 05862371

[24] G. Grubb, Extension theory for elliptic partial differential operators with pseudodifferential methods. For Proceedings of Leiden workshop 2009. Preprint 2008. arXiv:1008.1081

[25] G. Grubb and E. Schrohe, Trace expansions and the noncommutative residue for manifolds with boundary. J. reine angew. Math. 536 (2001), 167-207. MR 1837429 Zbl 0980.58017

[26] J. Johnsen, Pointwise multiplication of Besov and Triebel-Lizorkin spaces. Math. Nachr. 175 (1995), 85-133. MR 1355014 Zbl 0839.46026

[27] A. Laptev, Spectral asymptotics of a composition of pseudodifferential operators and reflections from the boundary. Dokl. Akad. Nauk SSSR 236 (1977), 800-830. English transl.: Soviet Math. Doklady 18 (1977), 1273-1276. MR 0454403 Zbl 0391.47035

[28] A. Laptev, Spectral asymptotics of a class of Fourier integral operators. Trudy Mosk. Mat. Obsv. 43 (1981), 92-115. English transl.: Trans. Moscow Math. Soc. 1 (1983), 101-127. MR 0651330 Zbl 0503.47044 (Russian version) Zbl 0535.47030 (English version) 
[29] J.-L. Lions and E. Magenes, Problèmes aux limites non homogènes et applications. Vol. I, Éditions Dunod, Paris, 1968. Zbl 0165.10801

[30] M. M. Malamud Spectral theory of elliptic operators in exterior domains. Russian J. Math. Phys. 17 (2010), 96-125. MR 2602538

[31] R. T. Seeley, Complex powers of an elliptic operator. AMS Proc. Symp. Pure Math. 10 (1967), 288-307 MR 0237943 Zbl 1202.35142

[32] E. Shamir, Mixed boundary value problems for elliptic equations in the plane. The $L^{p}$ theory. Ann. Scuola Norm. Sup. Pisa III 17 (1963), 117-139. MR 0157089 Zbl 0117.07002

Received September 6, 2010; revised December 30, 2010

Gerd Grubb, Department of Mathematical Sciences, Copenhagen University, Universitetsparken 5, 2100 Copenhagen, Denmark.

E-mail: grubb@math.ku.dk 